\title{
Carotid endarterectomy under local anesthesia: An institutional report of experience
}

\author{
Atilla Saraç ${ }^{1}$ (D) Artan Jahollari² (D) \\ ${ }^{1}$ Department of Cardiovascular Surgery, Medical Park Hospital, Samsun, Turkey \\ ${ }^{2}$ Department of Cardiovascular Surgery, American Hospital 3, Tirane, Albania
}

\begin{abstract}
Objectives: This study aims to present our personal single-surgeon experience regarding carotid endarterectomy (CEA) procedures performed under local anesthesia.

Patients and methods: Between January 2007 and December 2012, a total of 215 CEA procedures in 203 patients (164 males, 39 females; mean age $66.5 \pm 7.9$ years; range, 43 to 85 years) were retrospectively analyzed. Local anesthesia was achieved with single bolus injection of $10 \mathrm{~mL}$ of $2 \%$ prilocaine. An awake test was performed for three min following arterial clamping. Arteriotomy line was closed primarily in a continuous fashion. An intraluminal shunt was used in patients who developed neurological symptoms during the awake clamp testing.

Results: Twelve patients (5.9\%) underwent staged CEA for bilateral disease and 88 patients (43.3\%) underwent coronary artery bypass grafting following the initial CEA. None of procedures converted to general anesthesia. Transient ischemic attack developed in five patients (2.4\%), cerebral infarction in five patients (2.4\%), myocardial infarction in two patients (0.98\%), and a neck hematoma in six patients (2.9\%). Revision for neck hematoma within the first 24 hours was required in two patients (0.98\%). The mean follow-up was $40.1 \pm 6.6$ months. Two patients $(0.98 \%)$ had significant restenosis within two years and one of them underwent repeated CEA. There were five late mortalities (2.4\%). The patency rate was $99 \%$ during follow-up.

Conclusion: Low complication rates and excellent patency rates can be achieved using local anesthesia during CEA for isolated unilateral carotid stenosis. This technique also allows implementation of a staged procedure early after the operation.
\end{abstract}

Keywords: Carotid endarterectomy, carotid stenosis, local anesthesia.

Carotid endarterectomy (CEA) has been proven to be effective in the prevention of ischemic stroke in patients with symptomatic and asymptomatic carotid stenosis. Despite an increasing trend toward carotid stenting, CEA is still routinely performed due to large number of cases in the aging population. ${ }^{[1]}$ Although CEA is a uniform procedure involving complete elimination of the endarterectomy plaque and preservation of the remaining artery calibration, debates still continue regarding the use of an intraoperative shunt, technique for plaque removal and closure of the arteriotomy, and type of the anesthesia used (i.e., local or general). ${ }^{[2]}$

Carotid endarterectomy under local or locoregional anesthesia in the awake patients has long been used. ${ }^{[3]}$
Keeping the patient awake during surgery allows for assessing the neurological status during surgery, thereby, preventing unnecessary use of intraoperative shunts and related complications. ${ }^{[4]}$ Although not yet proven in randomized clinical trials, CEA under local anesthesia has been suggested to reduce perioperative morbidity and mortality in the latest reports. ${ }^{[5]}$

In this study, we present our personal singlesurgeon experiences regarding CEA procedures performed under local anesthesia.

\section{PATIENTS AND METHODS}

This retrospective study was conducted in Medical Park Hospital between January 2007 and

Received: December 27, 2019 Accepted: January 25, 2020 Published online: March 10, 2020

Correspondence: Artan Jahollari, MD. Rr: Sabaudin Gabrani, Nr. 2, Spitali Amerikan 3, Departamenti I kirurgjise Kardio-Vaskulare, 1000 Tirane, Albania. e-mail: artanjahollari@gmail.com 
December 2012. A total of 215 CEA procedures 203 patients (164 males, 39 females; mean age $66.5 \pm 7.9$ years; range, 43 to 85 years) were retrospectively analyzed. Patient counseling charts, laboratory and diagnostic test reports, and surgical reports were collected. Patients with neurological symptoms or carotid murmur were referred to Doppler ultrasound (DUS) and those having critical stenosis, subtotal or total occlusion in common and/or internal carotid artery underwent further investigation using contrastenhanced magnetic resonance imaging or computed tomography angiography. In addition, neurologically asymptomatic patients scheduled for elective coronary artery bypass grafting (CABG) were screened with carotid DUS and were evaluated further in the presence of $>70 \%$ stenosis. All symptomatic patients with $>50 \%$ stenosis and asymptomatic patients with $>70 \%$ were scheduled for CEA. Patients with a life expectancy of $<1$ year and those scheduled for repeated CEA were excluded. Preliminary neurological examination was performed to assess limb weakness, sensory loss, dysphagia, walking, and visual field. Baseline data including patient demographics, neurological symptoms, degree of carotid stenosis, and operative variables such as local anesthesia procedure, awake testing, internal carotid artery clamping time, intraoperative shunting, and surgical technique were recorded. Postoperative data consisted of early postoperative outcomes including transient ischemic attack, cerebral infarction, myocardial infarction (MI), tongue deviation, hematoma, voice changes and mortality rates. A written informed consent was obtained from each patient. The study protocol was approved by the Medical Park Hospital Ethics Committee. The study was conducted in accordance with the principles of the Declaration of Helsinki.

\section{Anesthesia and surgical procedure}

All patients received $0.01 \mathrm{mg}$ per $\mathrm{kg}$ of intravenous midazolam immediately before their transfer to the operating room. In the operating room, standard monitoring included electrocardiography, continuous blood pressure monitoring via radial artery catheterization, contralateral central venous line insertion, and pulse oximetry. The patient was laid in the supine position on the operating table adjusted at an angle of $45^{\circ}$ semi-upright position. After prepping and draping the surgical field, superficial and deep local anesthesia was achieved with a single bolus injection of $10 \mathrm{~mL}$ of $2 \%$ prilocaine.

The mean arterial pressure was kept close to the levels recorded before the operation. About $5 \mathrm{~min}$ before clamping, 5,000 IU of unfractionated heparin was given. The arterial clamps were, then, applied and an awake test was performed for three min. Cerebral flow monitoring with non-invasive methods was not used. An intraluminal shunt was used only, when the patient had symptoms of cerebral ischemia such as alteration in level of consciousness, seizures, slurred speech or motor deficit on the contralateral side of the body. A longitudinal arteriotomy incision was made
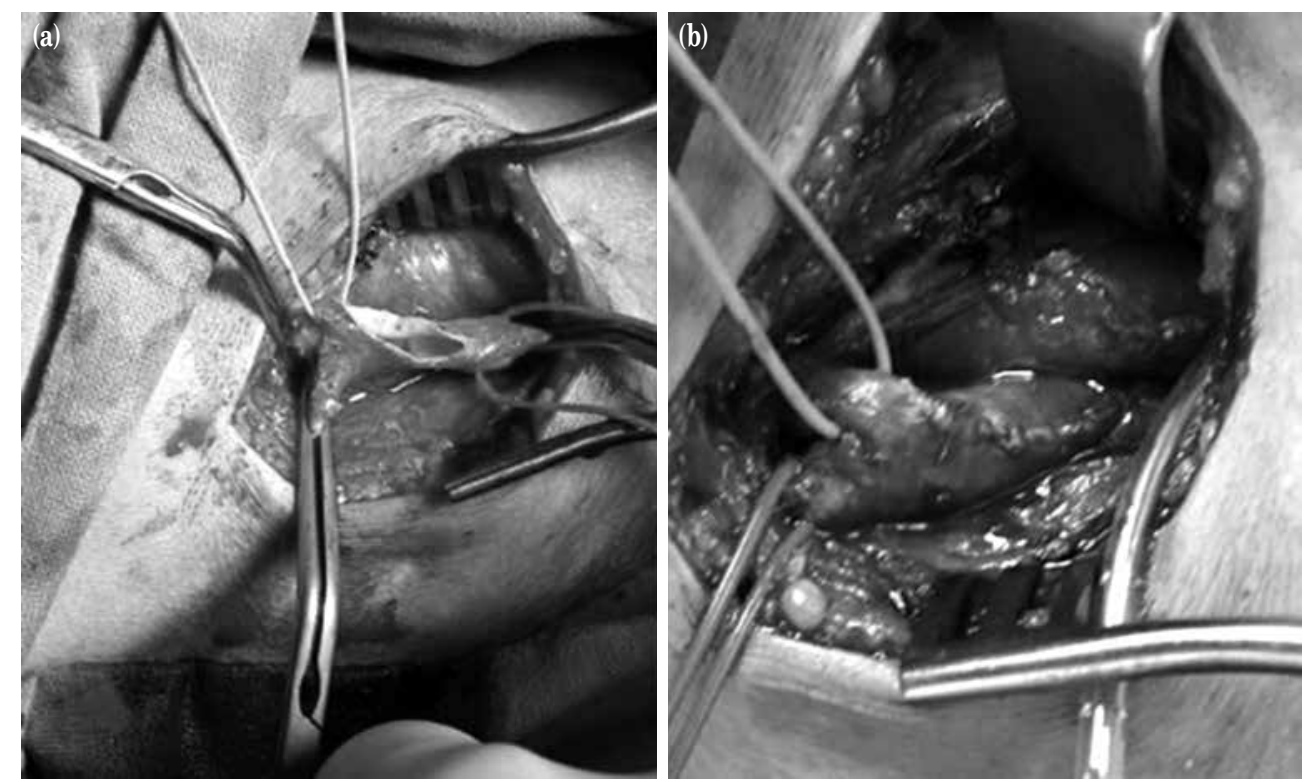

Figure 1. (a) Arteriotomy incision toward external carotid artery. (b) Primary saturation of arteriotomy line. 
over the common carotid artery and extended toward the internal carotid artery. When the diameter of the internal carotid artery was less than $5 \mathrm{~mm}$, arteriotomy incision was extended toward the external carotid artery (Figure 1a). The clamp over the internal carotid artery was temporarily removed during the extraction of the distal end of the plaque to achieve a complete endarterectomy and to remove distal debris (Figure 2). Arteriotomy line was closed primarily in a continuous fashion using a $7 / 0$ polypropylene suture (Figure $1 \mathrm{~b}$ ). A small drain was placed, and the layers were closed. All the patients received intravenous 5,000 IU heparin boluses four times a day, starting on the postoperative

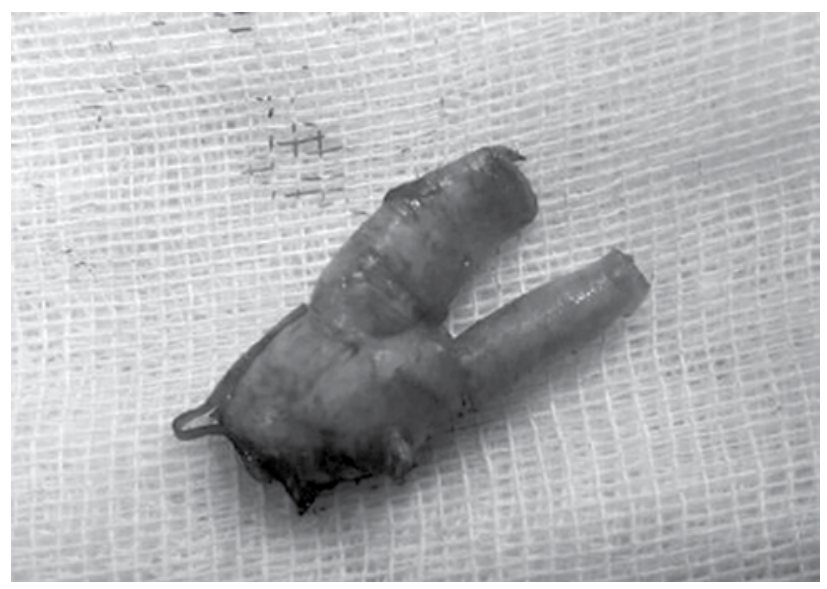

Figure 2. Complete endarterectomy achieved by distal unclamping during plaque extraction.

Table 1. Baseline demographic and clinical characteristics of patients

\begin{tabular}{lccc}
\hline Variable & $\mathrm{n}$ & $\%$ & Mean \pm SD \\
\hline Age (year) & & & $66.5 \pm 7.9$ \\
Male gender & 164 & 80.8 & \\
Diabetes mellitus & 93 & 45.8 & \\
Hypertension & 147 & 72.4 & \\
Smoking history & 153 & 75.3 & \\
Hyperlipidemia & 128 & 63.0 & \\
Stroke & 35 & 17.2 & \\
Symptomatic patients & 19 & 9.36 & \\
Chronic obstructive pulmonary disease & 23 & 11.3 & \\
Peripheral arterial disease & 33 & 16.2 & \\
Coronary artery disease & 95 & 46.8 & \\
Doppler ultrasound & 203 & 100 & \\
Magnetic resonance angiography & 23 & 11.3 & \\
Computed tomography angiography & 127 & 62.56 & \\
Catheter angiography & 53 & 26.1 & \\
\hline
\end{tabular}

SD: Standard deviation. sixth hour. In patients with bilateral carotid stenosis, contralateral endarterectomy was performed within one to three days. All patients were given dual antiplatelet therapy with clopidrogel $75 \mathrm{mg} /$ day and aspirin $150 \mathrm{mg} /$ day the day before discharge. Lipid lowering and antihypertensive medications were prescribed, as needed.

\section{Statistical analysis}

Statistical analysis was performed using the PASW version 17.0 software (SPSS Inc., Chicago, IL, USA). Continuous variables were expressed in mean \pm standard deviation (SD) and median (min$\max$ ), while categorical variables were expressed in number and frequency. The distribution of the variables was analyzed using the Kolmogorov-Smirnov test. Independent sample t-test and Mann-Whitney $\mathrm{U}$ test were used to analyze independent quantitative data. The chi-square test was used in the analysis of independent qualitative data. A $p$ value of $p<0.05$ was considered statistically significant.

\section{RESULTS}

Baseline characteristics of the patients are shown in Table 1. Among the patients who underwent isolated CEA ( $\mathrm{n}=115), 54(26.6 \%)$ had a history of previous stroke or transient ischemic attack and 61 (30.0\%) were asymptomatic. In addition, 88 patients were enlisted for a staged procedure consisting of $\mathrm{CEA}+\mathrm{CABG}$ and

\begin{tabular}{lcc} 
Table 2. Types of procedures performed & & \\
\hline Procedures & $\mathrm{n}$ & $\%$ \\
\hline Total patients & 203 & 100 \\
Total CEA & 215 & \\
Unilateral CEA & 191 & 94.0 \\
Bilateral staged CEA & 12 & 5.9 \\
Isolated CEA & 115 & 56.6 \\
CEA + CABG & 88 & 43.3 \\
\hline
\end{tabular}

CEA: Carotid endarterectomy; CABG: Coronary artery bypass grafting.

Table 3. Postoperative complications at one month

\begin{tabular}{lcc}
\hline Variable & $\mathrm{n}$ & $\%$ \\
\hline Transient ischemic attack & 5 & 2.4 \\
Stroke & 5 & 2.4 \\
Myocardial infarction & 2 & 0.98 \\
Death & 4 & 1.96 \\
Hematoma & 6 & 2.94 \\
Restenosis & 2 & 0.98 \\
Tongue deviation & 3 & 1.48 \\
Voice change & 2 & 0.98 \\
\hline
\end{tabular}


were screened among 1,634 patients scheduled for elective CABG.

The mean duration of clamping was $12.8 \pm 4.3 \mathrm{~min}$. An intraluminal shunt was used in six patients (2.9\%) who developed neurological symptoms during the awake clamp testing. Twelve patients (5.9\%) underwent staged CEA for bilateral disease and 88 patients (43.3\%) underwent CABG following the initial CEA. These staged operations were performed within one to three days after the initial CEA (Table 2). Patch angioplasty for closure of the arteriotomy was performed in one patient (1\%) due to calcified plaque formation extending quite distally. The frequency of postoperative complications was as follows: transient ischemic attack in five patients (2.4\%), cerebral infarction in five patients $(2.4 \%)$, MI in two patients (0.98\%), and neck hematoma in six patients (2.9\%). Revision for neck hematoma within the first 24 hours was required in two patients $(0.98 \%)$ (Table 3). There were four deaths (1.96\%) within 30-day of surgery: two from cardiac causes, one from stroke, and one from pulmonary complications.

The mean follow-up was $40.1 \pm 6.6$ (range, 22 to 58 ) months. Two patients $(0.98 \%)$ had significant restenosis within two years and one of them underwent reoperation CEA. There were five late deaths (2.4\%): one from cardiac disease, one from stroke, and three from unknown causes. The surviving patients remained neurologically asymptomatic throughout the follow-up period. The DUS examinations at six-month intervals demonstrated no restenosis (patency rate: 99\%) during follow-up.

\section{DISCUSSION}

This study presents our real-world experiences regarding CEA under local anesthesia. The technique resulted in low complication rates and favored a comfortable and time-saving procedure to the surgeon by reducing the unnecessary use of intraluminal shunts. According to the respective guidelines, the incidence of significant carotid stenosis in patients undergoing CABG ranges between 2.8 and $22 \% .^{[6-8]}$ In our study, 88 patients (5.38\%) were diagnosed with significant carotid stenosis among 1,634 patients who were scheduled for CABG during the study period.

To date, various techniques for CEA procedure have been described, such as primary closure or patch closure of the arteriotomy incision, and eversion method for removal of the plaque. Several studies have advocated patch closure to avoid stroke or restenosis, compared to primary closure. Rerkasem and Rothwell ${ }^{[9]}$ published a detailed review of the literature supporting the routine use of patch angioplasty after CEA. However, the trials included in this review were limited by small sample size and some of them had methodological shortcomings. In another review, Schwartz et al..$^{[8]}$ and Cao et al..$^{[10]}$ analyzed the results of studies comparing the outcomes of standard versus eversion CEA. The authors reported that, although less arterial occlusion and restenosis were observed in eversion CEA, these numbers were not translated into clinical benefits in terms of a reduced stroke risk.

A more recent study revealed superior hemodynamic features of primary closure compared to patch angioplasty, due to the presence of fewer areas of low wall shear stress and elevated oscillatory shear index. ${ }^{[1]}$ The authors of the study recommended the use of a trimmed patch $(5 \mathrm{~mm})$ rather than large ones, if necessary. However, another recent study from Turkey showed no difference regarding restenosis between the primary closure and patch angioplasty. ${ }^{[12]}$ In our routine practice, we also use primary closure method and our complication rates remain low.

Carotid endarterectomy can be performed under either local anesthesia or general anesthesia. The General Anesthesia Versus Local Anesthesia for Carotid Surgery (GALA) trial is the largest randomized surgical/anesthetic trial ever performed and included 3,526 patients recruited from 95 centers in 24 countries. ${ }^{[13]}$ This study found no significant difference between two types of anesthesia in terms of perioperative morbidity and mortality (particularly from stroke), quality of life in the short term, and strokeand MI-free survival at one year. ${ }^{[14]}$ In addition, better results were achieved in patients with contralateral carotid stenosis operated under local anesthesia, indicating a group that may benefit from a specific type of anesthesia. However, some methodological shortcomings of the GALA trial should be noted. First, the major advantage of local anesthesia during CEA is to avoid unnecessary intraluminal shunting. When neurological deterioration is observed during awake testing, a shunt can be placed to avoid cerebral ischemia, while the shunt itself may dislodge the atheroma plaque through the distal bed. Moreover, the shunt may result in intimal layer damage, which may possibly create a new source for intraoperative or early postoperative thrombosis. This situation may cause postoperative restenosis. In the GALA trial, the rate of shunt placement was $43 \%(n=738)$ and $14 \%(n=248)$ in general and local anesthesia groups, respectively. ${ }^{[8]}$ 
This inhomogeneity raises a suspicion: if the procedure would have been done under general anesthesia or local anesthesia and the topics of 'shunt preferred' or 'shunt not preferred' would have been investigated, the results could have been explained by protective or harmful effects of shunt usage in general anesthetic or local anesthetic approach. In the light of these results, it could have been indirectly reported that local anesthetic approach, decreasing shunt insertion, was better compared to general anesthesia. There are also similar criticisms on GALA study in the literature. ${ }^{[15,16]}$

Debate still continues over surgical treatment of concomitant carotid and coronary artery disease. A general opinion, supported by different studies, suggests that simultaneous CEA and CABG carry a cumulative complication risk and high cardiovascular event rates. On the contrary, a small study reported no increased operative risk for concomitant surgery. ${ }^{[17-19]}$ We routinely perform a staged approach and we often remove the carotid stenosis or occlusion first to avoid cerebral ischemia, unless cardiac deterioration occurs.

The non-randomized design and the lack of a control group are the main limitations of the study. In addition, this study presents single-surgeon experiences and the results documented may not be generalized.

In conclusion, our study results suggest that routine use of local anesthesia in CEA is a valid and effective modality, when the surgical team has gained enough experience with the technique. Low complication rates and excellent patency rates can be achieved by use of the technique in isolated unilateral carotid stenosis. The technique also allows for implementation of a staged procedure early after the operation. Combination of individualized treatment strategy and technical preferences of the surgeon are critical determinants to maintain low morbidity and mortality rates.

\section{Declaration of conflicting interests}

The authors declared no conflicts of interest with respect to the authorship and/or publication of this article.

\section{Funding}

The authors received no financial support for the research and/or authorship of this article.

\section{REFERENCES}

1. Lichtman JH, Jones MR, Leifheit EC, Sheffet AJ, Howard G, Lal BK, et al. Carotid Endarterectomy and Carotid Artery Stenting in the US Medicare Population, 19992014. JAMA 2017;318:1035-46.
2. Mantz F, Thibaud D, Munier P. Carotid surgery in private practice: what kind of change in the last 15 years? Ann Vasc Surg 2014;28:239-44.

3. Tangkanakul C, Counsell C, Warlow C. Local versus general anaesthesia for carotid endarterectomy. Cochrane Database Syst Rev 2000;2:CD000126.

4. Cho J, Lee KK, Yun WS, Kim HK, Hwang YH, Huh S. Selective shunt during carotid endarterectomy using routine awake test with respect to a lower shunt rate. J Korean Surg Soc 2013;84:238-44.

5. Hajibandeh S, Hajibandeh S, Antoniou SA, Torella F, Antoniou GA. Meta-analysis and trial sequential analysis of local vs. general anaesthesia for carotid endarterectomy. Anaesthesia 2018;73:1280-9.

6. Liapis CD, Bell PR, Mikhailidis D, Sivenius J, Nicolaides A, Fernandes e Fernandes J, et al. ESVS guidelines. Invasive treatment for carotid stenosis: indications, techniques. Eur J Vasc Endovasc Surg 2009;37:1-19.

7. Hertzer NR, Young JR, Beven EG, Graor RA, O'Hara PJ, Ruschhaupt WF 3rd, et al. Coronary angiography in 506 patients with extracranial cerebrovascular disease. Arch Intern Med 1985;145:849-52.

8. Schwartz LB, Bridgman AH, Kieffer RW, Wilcox RA, McCann RL, Tawil MP, et al. Asymptomatic carotid artery stenosis and stroke in patients undergoing cardiopulmonary bypass. J Vasc Surg 1995;21:146-53.

9. Rerkasem K, Rothwell PM. Systematic review of randomized controlled trials of patch angioplasty versus primary closure and different types of patch materials during carotid endarterectomy. Asian J Surg 2011;34:32-40.

10. Cao PG, de Rango P, Zannetti S, Giordano G, Ricci S, Celani MG. Eversion versus conventional carotid endarterectomy for preventing stroke. Cochrane Database Syst Rev 2001;1:CD001921.

11. Harrison GJ, How TV, Poole RJ, Brennan JA, Naik JB, Vallabhaneni SR, et al. Closure technique after carotid endarterectomy influences local hemodynamics. J Vasc Surg 2014;60:418-27.

12. İyigün T, Kyaruzi M, Timur B, İyigün M, Aydın Ü. Our midterm restenosis results using patch angioplasty closure versus primary closure in patients undergoing carotid endarterectomy: A comparative study. Turk J Vasc Surg 2019;28:19-23.

13. GALA Trial Collaborative Group, Lewis SC, Warlow CP, Bodenham AR, Colam B, Rothwell PM, et al. General anaesthesia versus local anaesthesia for carotid surgery (GALA): a multicentre, randomised controlled trial. Lancet 2008;372:2132-42.

14. Gough MJ, Bodenham A, Horrocks M, Colam B, Lewis SC, Rothwell PM, et al. GALA: an international multicentre randomised trial comparing general anaesthesia versus local anaesthesia for carotid surgery. Trials 2008;9:28.

15. Guay J. The GALA trial: answers it gives, answers it does not. Lancet 2008;372:2092-3.

16. Gough MJ; GALA Trial Collaborators. The GALA Trial-a summary of the findings. Eur J Vasc Endovasc Surg 2008;36:505-6.

17. Naylor AR. Does the risk of post-CABG stroke merit staged or synchronous reconstruction in patients with 
symptomatic or asymptomatic carotid disease? J Cardiovasc Surg 2009;50:71-81.

18. Prasad SM, Li S, Rankin JS, O’Brien SM, Gammie JS, Puskas JD, et al. Current outcomes of simultaneous carotid endarterectomy and coronary artery bypass graft surgery in
North America. World J Surg 2010;34:2292-8.

19. Ren S, Liu P, Ma G, Wang F, Qian S, Fan X. Long-term outcomes of synchronous carotid endarterectomy and coronary artery bypass grafting versus solely carotid endarterectomy. Ann Thorac Cardiovasc Surg 2012;18:228-35. 